\title{
Comparison of Compression Algorithms' Impact on Iris Recognition Accuracy
}

\author{
Stefan Matschitsch ${ }^{1}$, Martin Tschinder ${ }^{1}$, and Andreas Uhl ${ }^{1,2}$ \\ ${ }^{1}$ School of Telematics \& Network Engineering, Carinthia Tech Institute, Austria \\ ${ }^{2}$ Department of Computer Sciences, Salzburg University, Austria \\ uhl@cosy.sbg.ac.at
}

\begin{abstract}
The impact of using different lossy compression algorithms on the matching accuracy of iris recognition systems is investigated. In particular, we relate rate-distortion performance as measured in PSNR to the matching scores as obtained by a concrete recognition system. JPEG2000 and SPIHT are correctly predicted by PSNR to be well suited compression algorithms to be employed in iris recognition systems. Fractal compression is identified to be least suited for the use in the investigated recognition system, although PSNR suggests JPEG to deliver worse recognition results in the case of low bitrates. PRVQ compression performs surprisingly well given the third rank in PSNR performance, resulting in the best matching scores in one scenario. Overall, applying compression algorithms is found to increase FNMR but does not impact FMR. Consequently, compression does not decrease the security of iris recognition systems, but "only" reduces user convenience.
\end{abstract}

\section{Introduction}

With the increasing usage of biometric systems the question arises naturally how to store and handle the acquired sensor data. In this context, the compression of these data may become imperative under certain circumstances due to the large amounts of data involved. Among other possibilities (e.g. like template storage on IC cards), compression technology may be used in two stages of the processing chain in classical biometric recognition:

1. Storage of reference data: In most template databases (where the reference data of the enrolled individuals is stored) only the extracted features required for the matching step are stored as opposed to retaining the originally acquired sensor data. However, in case the features should be replaced for some reason (e.g. when a superior or licence-free matching technique involving a different feature set becomes available), having stored only extracted features implies the requirement for all legitimate users for a re-enrollment, which can be expensive and is highly undesired since user-acceptance of the entire biometric system will suffer. Storing the original sensor data in addition to the features required for the current matching technique solves this problem. Of course, these data need to be stored in compressed (to save storage space) and encrypted (to protect privacy) form. 
2. Transmission of sample data after sensor data acquisition: In distributed biometric systems, the data acquisition stage is often dislocated from the feature extraction and matching stage (this is true for the enrollment phase as well as for authentication). In such environments the sensor data have to be transferred via a network link to the respective location, often over wireless channels with low bandwidth and high latency. Therefore, a minimization of the amount of data to be transferred is highly desirable, which is achieved by compressing the data before transmission. An alternative solution would be to extract the features before transmission and to transfer feature data only - in many cases, feature extraction is more demanding as compared to compression which generates additional workload for the often mobile and low power acquisition devices.

Having found that compression of the raw sensor data can be advantageous in certain applications, we have to identify techniques suited to accomplish this task in an optimal manner. In order to maximize the benefit in terms of data reduction, lossy compression techniques have to be applied. However, the distortions introduced by compression artifacts may interfere with subsequent feature extraction and may degrade the matching results. In particular, FRR or FNMR will increase (since features of the data of legitimate users are extracted less accurately from compressed data) which in turn affects user convenience and general acceptance of the biometric system. In extreme cases, even FAR or FMR might be affected.

In this work, we will focus on the compression of iris images. Contrasting to the overwhelming majority of literature and studies in the field of compressing biometric sample data, we will not rely on assessing the resulting objective and subjective image quality after compression only, but we will apply a biometric iris recognition systems to the compressed sensor data to evaluate the effects of compression on recognition accuracy, in particular on the matching results of legitimate and illegitimate users.

In Section 2, we will review and discuss the available literature on biometric sample data compression with focus on iris data storage. Section 3 is the main part of this work where we first describe the employed image compression techniques, the used iris recognition system, and the data these algorithms are applied to. Subsequently we present and discuss our experimental results where we compare and rank the compression algorithms in use. Section 4 concludes the paper.

\section{Iris Image Compression}

The reason for focussing our investigations to iris recognition systems is that this biometric modality is claimed to be the most secure one exhibiting practically $0 \%$ FAR and low FRR. An interesting fact is that the iris recognition market is strongly dominated by Iridian Inc. based technology which is based on algorithms of J. Daugman [11]. The certainly most relevant standard for 
compressing iris image data is the recent ISO/IEC 19794-6 standard on Biometric Data Interchange Formats.

While the data formats specified by the ISO/IEC 19794 standard are fixed at present state, their impact on recognition accuracy as compared to other algorithms is not well documented. This is the scope of the current paper.

A vast amount of literature exists in which different compression algorithms are compared with respect to their rate-distortion performance on "usual" image data. It is worth noticing that also the evaluation of compression performance in biometric systems is surprisingly limited to PSNR or RMS-error computation and to psycho-visual studies in most investigations. Effects on actual matching rates or recognition accuracy are rarely documented. One of the few exceptions is found in 13 where image tiles containing fingerprint minutiae are compressed using PCA and wavelet transform and the effects on FAR of a specific recognition system are studied. In recent work [8], we have investigated the impact of JPEG, JPEG2000, SPIHT, PRVQ, and Fractal image compression on recognition accuracy of selected fingerprint and face recognition systems. 4 also relates JPEG, JPEG2000, and (WSQ) compression rates to recognition performance of some fingerprint and face recognition systems. Compression effects with respect to face recognition accuracy have been studied in dedicated papers as well: 6] investigates the impact of JPEG and JPEG2000 on the recognition results of 12 different face recognition techniques, and [7] employs varying bitrates using JPEG2000 to determine the trade-off points between recognition accuracy and compression rate.

ISO/IEC 19794-6 allows iris image data to be stored in lossy manner in the JPEG and JPEG2000 formats. Two types of iris image data are considered: rectilinear images (i.e. images of the entire eye) and polar images (which are basically the result of iris detection and segmentation), the latter much smaller in terms of storage requirement (e.g. $2 \mathrm{kB}$ vs. $25-30 \mathrm{kB}$ for rectilinear images). It is important to note that with this standardization it might become more attractive for companies to investigate alternatives to Iridian products due to the available common data format iris recognition systems can rely on.

Few studies are available on iris image compression and its impact on recognition performance. [10] applies JPEG2000 up to a compression rate of 20 to rectilinear image data (the CASIA database and a proprietary image collection is used) and investigates the effects on FAR and FRR of a 1-D version of the Daugman algorithm (the same system which is used in this study). 9] again uses JPEG2000 to compress polar iris images up to a compression rate of 80 and studies the impact on verification accuracy of three iris recognition systems (including the Daugman algorithm, the CASIA database is used). A more compact way of representing the Daugman IrisCode is discussed in [12, however, these results refer to template compression and are only valid for the techniques related to Iridian products.

In the subsequent experimental study we will apply a set of general purpose compression algorithms to rectangular iris image data and we will study their respective impact on the recognition accuracy of an iris template matching 
technique. Contrasting to the two studies reviewed before, we will not only rely on assessing JPEG2000 performance but will also investigate the performance of other schemes, e.g. like JPEG which is also covered by ISO/IEC 19794-6. Specifically, we will relate the rate-distortion performance of the compression schemes to the matching score of the recognition system applied to the compressed data. In this way, we are able to compare the compression schemes for their respective usefulness in the biometric context.

\section{Experimental Study}

\subsection{Setting and Methods}

Compression Algorithms. We use five different general purpose image compression algorithms: JPEG [1], JPEG2000[2], SPIHT [5], FRAC[14] and PRVQ 3]. Since the block-DCT based JPEG standard is a part of almost any image processing tool we do not give further reference on it. For the wavelet-based JPEG2000 standard we have used the JAVA reference implementation JJ20001. SPIHT relies as well on the wavelet transform, but contrasting to JPEG2000 which exploits intra subband correlations only, SPIHT is a zero-tree based codec which exploits coefficient dependencies across subbands. We have employed the implementation as provided by the developers originally2. In addition to these described three transform-based compression algorithms we also use codebook-based schemes. Fractal image compression (FRAC) exploits self similarities within images (where the image itself may be interpreted as an internal codebook) and has been discussed controversially in the literature. We have employed an adaptive quadtree method implementation 3 based on Y. Fishers code. As the fifth algorithm a special flavour of vector quantization is used. PRVQ4 performs a block-based prediction within the image to be compressed (similar to the intraprediction mode in H.264), subsequently the residual image is compressed by a vector quantization strategy. [3] describes PRVQ and the used fractal compression variant in some detail. We apply each compression algorithm with 12 different bitrates distributed over the sensible operation range.

When applied to common images, JPEG2000 and SPIHT usually give the best results in terms of PSNR, closely followed by PRVQ. For high bitrates, JPEG provides fourth best results, whereas FRAC is superior to JPEG for medium and low bitrates. We expect the best results from JPEG2000, SPIHT and PRVQ, whereas JPEG and FRAC may not be able to represent the fine structure of the iris images with higher compression rates.

Iris Recognition System. The employed iris recognition system is Libor Masek's Matlab implementation [5] of a 1-D version of the Daugman iris

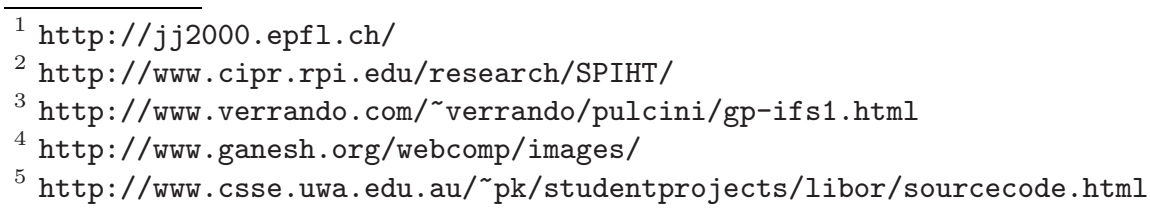


recognition algorithm. First, this algorithm segments the eye image into the iris and the remainder of the image. After extracting the features if the iris (which are strongly quantized phase responses of complex 1-D Gabor filters in this case), considering translation, rotations and disturbed regions in the iris (a noise mask is generated), the algorithm outputs the similarity score by giving the hamming distance between two extracted templates. The range of the hamming distance reaches from zero (ideal matching of two iris images of the same person) to 0.5 (ideal mismatch between two iris images of different persons).

Sample Data. For all our experiments we considered images with 8-bit grayscale information per pixel from the CASIA 61.0 iris image database (all images had been cropped and stored in a quadratic shape with a size of 280x280 pixels). We applied the experimental calculations on the images of the first 20 persons in the CASIA database using 7 iris images of each person. All employed compression software is able to handle this type of imagery, which is not true for color or non-squared images, and was applied on the raw rectangular image data and not on the extracted features. This has an important implication on the performance of the entire system. Whereas in the case of compressing polar iris images [9] only the iris texture information is affected, in the case of compressing rectangular image data also the iris detection and determination of the noise mask is potentially affected in addition to degrading texture information. Figure 1 shows an example of a JPEG2000 compressed (compression rate 96) iris image of one person, which was used in our calculations together with the extracted iris template data and the noise masks (template and noise mask have been scaled in y-direction by a factor of 4 for proper display).
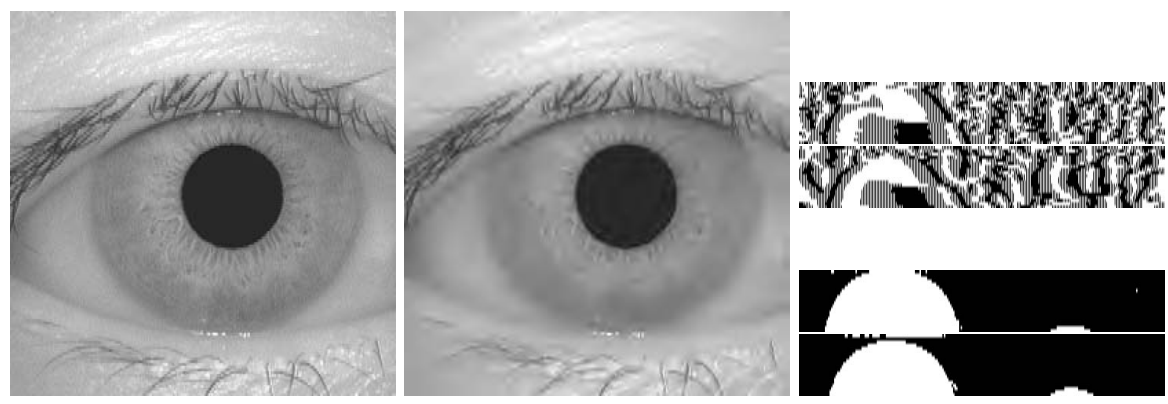

Fig. 1. Comparison of uncompressed/compressed iris image and the corresponding iris templates and noise masks

The differences in the templates are more significant as those in the noise masks which turn out to be very similar. Overall also iris detection is very robust to compression - all matching results shown in the following section have been generated without the software failing to detect the iris (results for compression

${ }^{6}$ http://www. sinobiometrics.com 
rates where this happens have been omitted as found with fractal compression for example).

Compression can be used in various stages of the matching process. Either the stored reference data may be in compressed form, or the sample data acquired for verification may be compressed (e.g. for efficient transfer), or both. Therefore, we use two settings in our experiments: either both images are compressed and matched against each other or only one image is compressed in the matching stage. For investigating correct matches (matches from legitimate users enrolled in the database), we rely on 172800 generated images (i.e. for each of the 20 persons, we have 7 images resulting in 6 ! possible correct matches each; considering the 12 different compression rates we finally result in $20 \cdot 6 ! \cdot 12=172800$ ) for each compression technique. This is only true in the scenario with only 1 compressed image, for 2 compressed images this number is reduced due to symmetry reasons. For investigating matches between different persons (imposter matches), far more data is available.

\subsection{Experimental Results}

Figure 2 shows the averaged rate distortion comparison of the different compression algorithms applied to all iris images considered.

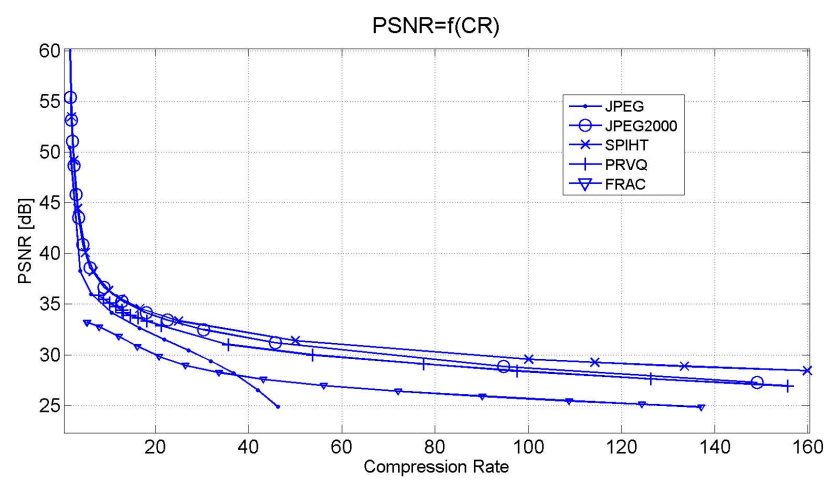

Fig. 2. Comparison of rate distortion of different compression algorithms

The highest average PSNR value is reached by JPEG2000 and SPIHT. PRVQ reaches PSNR values which are very close to those of JPEG2000 and SPIHT. JPEG and FRAC show the worst PSNR behaviour, which leads to the assumption that JPEG2000, SPIHT and PRVQ could be most suitable in iris recognition systems. Interestingly, FRAC outperforms JPEG for compression rates $>36$. In the following, we investigate the impact of compression on the matching score (i.e. obtained hamming distance (HD)). The interval of $0.26 \leq H D \leq 0.35$ is discussed as the border between match and mismatch in iris recognition [11 - based on recommendations for the specific technique 10 used we choose $H D=0.32$ as decision criterion between match and mismatch. 
Fig. 3 shows the plot of the HD after applying the iris recognition algorithm to the JPEG compressed iris images. The $\mathrm{x}$-axis shows the compression rates, whereas the y-axis shows the averaged hamming distance as well as the mean standard deviation of the HD.

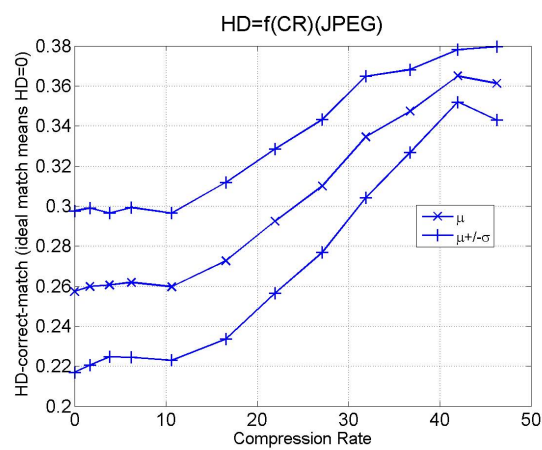

(a) legitimate users

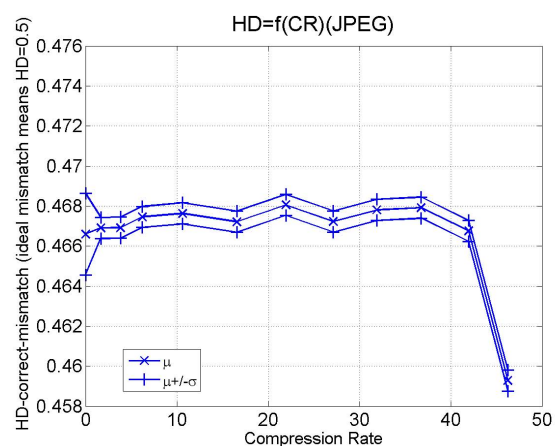

(b) imposters

Fig. 3. Impact of JPEG compression on iris recognition, 2 compressed images

In the case of legitimate users (Fig. 3. a), the mean value of the HD stays constant at approximately 0.26 until the compression rate exceeds 10 . The mean standard deviation is approximately 0.04. A further increase of the compression rate leads to a steady increase of HD and crosses the matching threshold of 0.32 at a compression rate of 28 . Note that this refers to averaged $H D$ values which implies the occurrence of a significant number of false negative matches at this compression rate.

For the case of imposters (irises from different persons are matched against each other - Fig. 3. b) the HD remains above 0.45 across the whole range of compression rates and deviation is low $(<0.01)$, which means that JPEG compression does not introduce any false positive matches.

The general trend of the other compression schemes is almost identical with respect to standard deviations and concerning the absence of false positive matches in the case of imposters. Therefore, these issues are not investigated further. In the following, we compare the averaged HD values among the different compression techniques for the case of correct matches - legitimate users. Figs. 4(a) and 4(b) compare the scenarios with 1 and 2 compressed image(s).

The results indicate that PSNR is in most cases a good predictor for matching performance with compressed iris images. But there are some subtle differences between PSNR results and average HD matching scores. Even though PSNR values suggest FRAC to be superior to JPEG for compression rate $>36$, there are no matching scores reported for FRAC in case of rate $>20$. The reason is that for higher compression rates the iris detection process fails and no sensible results are obtained. As suggested by their respective PSNR values, JPEG2000, SPIHT, and PRVQ perform similarly crossing the 0.32 border at a compression rate of about $80-90$. The superiority of those three compression 


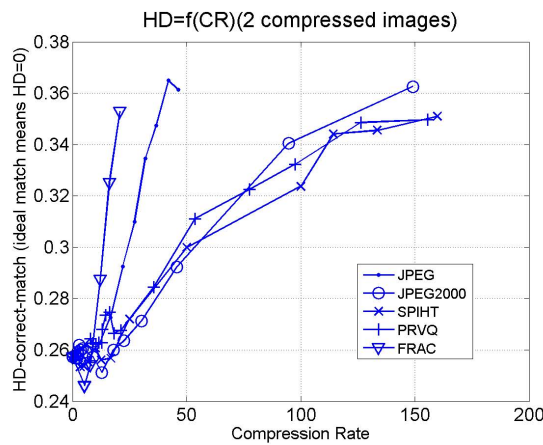

(a) 2 compressed images

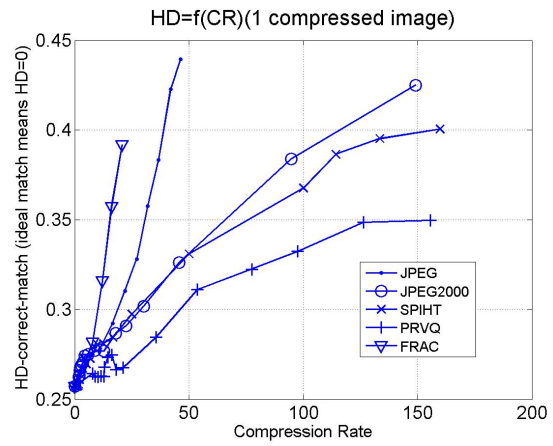

(b) 1 compressed image

Fig. 4. Comparison of the HD for iris images for legitimate users

techniques over JPEG in terms of HD is even more significant as suggested by PSNR. When comparing the scenarios with 1 or 2 compressed image(s), it immediately gets clear that much lower HD scores are obtained in the 2 compressed images case. When considering the 1 compressed image case (Fig. 4(b)), only PRVQ shows matching scores similar to the 2 compressed images case, all other compression schemes perform worse. It is interesting that PRVQ is clearly the best algorithm is this scenario although only ranked third in terms of PSNR. Given the fact that lossy compression also acts as some sort of denoising, it is not surprising that the 2 compressed images scenario delivers lower HD values. The claim that compression up to a rate of 16 even improves the matching scores of not compressed images 9] can be supported at least for the 2 compressed images case.

In order to get rid of the standard deviation in the comparisons and to use a quantity often employed in the assessment of biometric system performance, we compute the false non-match rate (FNMR):

$$
F N M R=\frac{\text { Number of (false) negative matches }}{\text { Number of legitimate users'matches }} .
$$

Figs. 5(a) and 5(b) compare the FNMR of different compression algorithms as a function of compression rate.

We notice similar behaviour as when comparing averaged HD values if we consider the ranking of the algorithms and when comparing the 1 and 2 compressed image(s) scenarios (the 2 compressed image scenario results in lower FNMR). Here, PRVQ is worse compared to JPEG2000 and SPIHT in the 2 compressed images case. This suggests the existence of more statistical outliers as for the other techniques leading to a higher number of false negative matches. Once again we would like to point out the performance difference between JPEG and JPEG2000: whereas JPEG reaches a FNMR of 50\% at a compression rate of 20, JPEG2000 attains the same value at a compression rate of 40. However, for lower bitrates and more realistic FNMR values, the difference is not that pronounced but still significant. 


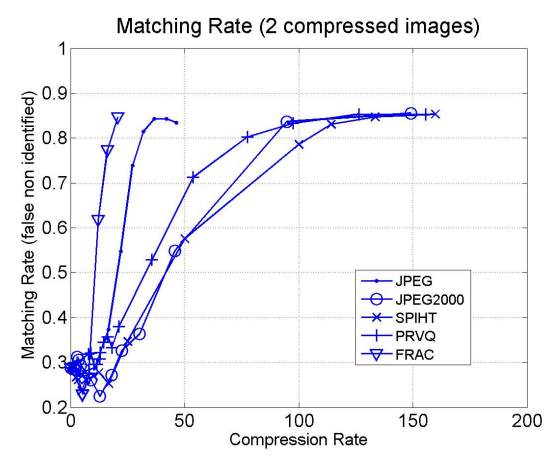

(a) 2 compressed images

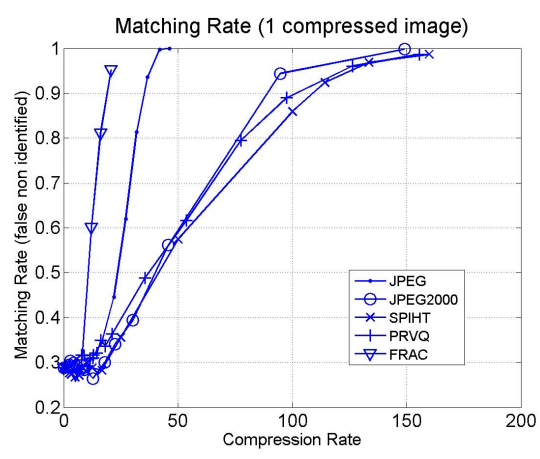

(b) 1 compressed image

Fig. 5. FNMR with decision threshold at 0.32

Finally, we take a look on FMR. FMR is $0 \%$ in the case of 1 compressed image for all algorithms. In the case of the comparison of 2 compressed images, we found one false positive match at a compression rate of 130 with SPIHT - but this outlier is not a serious problem, because a compression rate of 130 is much too high for a realistic FNMR (see Figures 5(b) and 5(a) and so an iris recognition system will never be operated at such high compression rates.

\section{Conclusion and Future Work}

JPEG2000, SPIHT, and PRVQ have been shown to be almost equally well suited for iris image compression. JPEG is significantly inferior to those techniques, especially when the bitrate decreases, the FNMR goes up dramatically fast. Fractal compression can be said to be non-suited at all for this application due to failing iris detection for compression rates $>20$. Scenarios involving the compression of both images involved in the matching process turn out to deliver better matching scores and lower FNMR as compared to the case of compressing only 1 image. Therefore, in case compression is employed, both reference as well as sample data should be compressed. Overall, applying compression algorithms is found to increase FNMR but does not impact FMR. Consequently, compression does not decrease the security of iris recognition systems, but "only" reduces user convenience. Finally, it should be noted that the differences among the compression algorithms are relatively small with respect to recognition accuracy in a high quality environment with compression ratios $<10$.

\section{Acknowledgements}

Most of the work described has been done in the scope of a semester project in the master program "Communication Engineering for IT" at CTI, Klagenfurt. 


\section{References}

[1] Pennebaker, W., Mitchell, J.: JPEG - Still image compression standard. Van Nostrand Reinhold, New York (1993)

[2] Taubman, D., Marcellin, M.: JPEG2000 — Image Compression Fundamentals, Standards and Practice. Kluwer Academic Publishers, Dordrecht (2002)

[3] Jerabek, B., Schneider, P., Uhl, A.: Comparison of lossy image compression methods applied to photorealistic and graphical images using public domain sources. Tech. Rep. RIST++15/98, Research Institute for Softwaretechnology, University of Salzburg (1998)

[4] Funk, W., Arnold, M., Busch, C., Munde, A.: Evaluation of image compression algorithms for fingerprint and face recognition systems. In: Cole, J., Wolthusen, S. (eds.) Proceedings from the Sixth Annual IEEE Systems, Man and Cybernetics (SMC) Information Assurance Workshop, June 2006, pp. 72-78. IEEE Computer Society, Los Alamitos (2006)

[5] Said, A., Pearlman, W.A.: A new, fast, and efficient image codec based on set partitioning in hierarchical trees. IEEE Transactions on Circuits and Systems for Video Technology 6, 243-249 (1996)

[6] Delac, K., Grigic, M., Grigic, S.: Effects of JPEG and JPEG2000 compression on face recognition. In: Singh, S., Singh, M., Apte, C., Perner, P. (eds.) ICAPR 2005. LNCS, vol. 3687, pp. 136-145. Springer, Heidelberg (2005)

[7] McGarry, D., Arndt, C., McCabe, S., D'Amato, D.: Effects of compression and individual variability on face recognition performance. In: Biometric Technology for Human Identification. Proceedings of SPIE, vol. 5404, pp. 362-372 (August 2004)

[8] Mascher-Kampfer, A., Stögner, H., Uhl, A.: Comparison of Compression Algorithms' Impact on Fingerprint and Face Recognition Accuracy. In: Visual Computing and Image Processing VCIP 07. Proceedings of SPIE, vol. 6508, pp. 65080N1-65050N-10 (January 2007)

[9] Rakshit, S., Monro, D.M.: Effects of Sampling and Compression on Human Iris Verification. In: Proceedings of the IEEE International Conference on Acustics, Speech, and Signal Processing ICASSP 2006. IEEE Signal Processing Society, pp. II-337-II-340 (July 2007)

[10] Ives, R.W., lBonney, B.L., Etter, D.M.: Effect of image compression on iris recognition. In: IMTC 2005 - Instrumentation and Measurement Technology Conference (May 2005)

[11] Daugman, J.: How iris recognition works. IEEE Transactions on Circiuts and Systems for Video Technology 14(1), 21-30 (2004)

[12] von Seelen, U.: IrisCode template compression and its effects on authentication performance. In: Biometrics Consortium Conference 2003 (September 2003)

[13] Beleznai, C., Ramoser, H., Wachmann, B., Birchbauer, J., Bischof, H., Kropatsch, W.: Memory-efficient fingerprint verification. In: Proceedings of the IEEE International Conference on Image Processing (ICIP'01), Thessaloniki, Greece, vol. 2, pp. 463-466 (October 2001)

[14] Fisher, Y. (ed.): Fractal Image Compression: Theory and Application. Springer, New York (1995)

[15] Masek, L., Kovesi, P.: MATLAB Source Code for a Biometric Identification System Based on Iris Patterns. The School of Computer Science and Software Engineering, The University of Western Australia (2003) 